\title{
Electrical Interfacial Layer at Hematite/Dicarboxylic Acid Aqueous Interface ${ }^{\dagger}$
}

\author{
Jasmina Salopek, Nikola Kallay, and Davor Kovačević* \\ Division of Physical Chemistry, Department of Chemistry, Faculty of Science, University of Zagreb, \\ Horvatovac 102a, HR-10000 Zagreb, Croatia
}

RECEIVED SEPTEMBER 21, 2012; REVISED OCTOBER 30, 2012; ACCEPTED NOVEMBER 28, 2012

\begin{abstract}
The interfacial properties of the system hematite/dicarboxylic acid were investigated in the presence of sodium perchlorate by means of adsorption, zeta potential and surface potential measurements. Maleic and fumaric acid were used as dicarboxylic acids. No significant difference in the surface potential values of hematite with and without the presence of dicarboxylic acids was observed. On the other hand, the zeta potential and the corresponding isoelectric point values obtained after adsorption of fumaric and maleic acids on hematite differ significantly from the zeta potential values of pure hematite. The parameters characterizing the electrical interfacial layer were determined on the basis of simultaneous interpretation of experimental data showing that there are some differences in adsorption behavior of two investigated dicarboxylic acids. The obtained equilibrium constant for adsorption of fumaric acid $\left(\log K^{\circ}=4.9 \pm 0.2\right)$ is higher than the same constant obtained for adsorption of maleic acid $\left(\log K^{\circ}=4.0 \pm 0.1\right)$ and the value of $C_{1}$ was in both cases determined as $2.2 \pm 0.3 \mathrm{~F} \mathrm{~m}^{-2}$. (doi: $10.5562 / \mathrm{cca} 2176$ )
\end{abstract}

Keywords: hematite, maleic acid, fumaric acid, adsorption, zeta potential, surface potential

\section{INTRODUCTION}

The study of adsorption of organic acids on metal oxide and other mineral surfaces is important both from the fundamental and from the application point of view having implications in various fields such as soil chemistry, geochemistry and waste water treatment. Among other organic acids, dicarboxylic acids are particularly interesting because they arise as waste products from several industrial processes. Interaction of fumaric and maleic acids in aqueous solution with synthetic hydroxyapatite was studied by Vega and Colinas. ${ }^{1}$ They found that both acids are adsorbed to hydroxyapatite via the completely deprotonated carboxylates, but that maleic acid shows stronger adsorption due to its cis geometry. They concluded that hydroxyapatite could be applied for selective removal of fumaric and maleic acids from wastewaters. Persson and coworkers ${ }^{2}$ studied the adsorption of dicarboxylates (among them of maleate) on nano-sized gibbsite particles by means of quantitative batch adsorption experiments and ATR-FTIR spectroscopy. The main aim of their study was to identify the molecular level bonding mechanisms of the dicarboxylates to gibbsite. They concluded that carboxylates with $z=-2$ ( $z$ being the charge number) formed predominantly outer sphere complexes, whereas the importance of inner sphere complexes progressively increased for $z=-1$ and $z=0$. The inner sphere structures were identified as mononuclear chelates with one oxygen from each carboxylate group bonded to $\mathrm{Al}(\mathrm{III})$ at the surface. On the other hand, Yao and $\mathrm{Yeh}^{3}$ studied the adsorption of fumarate, maleate and succinate on hydrous $\mathrm{Al}_{2} \mathrm{O}_{3}$ and observed that the adsorption of $\mathrm{HX}^{-}$ is more favorable than adsorption of $\mathrm{H}_{2} \mathrm{X}$ or $\mathrm{X}^{2-}$ and that the adsorption maximum of maleate was about $10 \%$ higher than that of fumarate. Hwang and Lenhart ${ }^{4}$ also investigated hematite/maleic acid system and they concluded on the basis of ATR-FTIR and batch adsorption experiments that both outer-sphere and inner-sphere complexes are simultaneously present, with inner-sphere complexes being favored at low $\mathrm{pH}$. On the other hand, their results suggest that in the case of adsorption of fumaric acid on hematite surface only outer-sphere complexes are formed. Finally, their results show that subtle differences in the structure of adsorbed acids produce important differences in the physicochemical behavior of particles in dilute aquatic systems.

From the above mentioned studies it is obvious that the equilibrium at various solid-liquid interfaces is very often a subject of both experimental and theoretical

\footnotetext{
$\dagger$ This article belongs to the Special Issue devoted to the $85^{\text {th }}$ anniversary of Croatica Chemica Acta.

* Author to whom correspondence should be addressed. (E-mail: davor.kovacevic@chem.pmf.hr)
} 
investigations, but various uncertainties still exist. In many cases the interpretation of surface equilibria is based on only one type of experimental data (e.g. only adsorption data) and such an approach may lead to erroneous conclusions regarding the mechanism of binding and the structure of the Electrical Interfacial Layer (EIL). We have already shown earlier ${ }^{5-7}$ that the introduction of other, additional experimental techniques could provide useful information leading to the more accurate determination of various adsorption parameters, but also to valuable indications about the location of adsorbed species within the EIL and to the binding mechanism. In this article we use such an approach for comparison of parameters obtained in the case of adsorption of two dicarboxylic acids (fumaric and maleic acid) on hematite. For that purpose we performed adsorption, zeta potential and, recently introduced, surface potential measurements by means of single crystal electrodes. ${ }^{8}$ Therefore we decided to examine these differences using abovementioned methods and have applied simultaneous interpretation of data.

\section{THEORETICAL}

When discussing the models that describe the electrical interfacial layer at the solid/liquid interface the number of postulated layers, i.e., planes that divide these layers and corresponding potentials should be assumed. In the so-called general model three layers and four different planes (with corresponding potentials) are postulated. ${ }^{9}$ The interpretation of experimental results presented in this paper is based on the measured surface $\left(\Psi_{0}\right)$ and electrokinetic zeta $(\zeta)$ potentials. Surface potential is the potential at the (inner) surface plane, 0-plane. In this plane the charged species are directly bound to the surface. On the other hand, the electrokinetic $\zeta$-potential corresponds to the imaginary slip or shear plane that is located within the diffuse layer and close to the d-plane which is assumed to be the onset of diffuse layer. According to the Gouy-Chapman theory, the relationship between the potential at the onset of diffuse layer and the electrokinetic $\zeta$-potential is given by:

$$
\Psi_{\mathrm{d}}=\frac{2 R T}{F} \ln \left(\frac{\exp \left(-\kappa l_{\mathrm{e}}\right)+\tanh (F \zeta / 4 R T)}{\exp \left(-\kappa l_{\mathrm{e}}\right)-\tanh (F \zeta / 4 R T)}\right)
$$

where $l_{\mathrm{e}}$ is the distance between d-plane and slip plane, corresponding to the thickness of electrokinetic stagnant layer, while $\kappa$ is the Debye-Hückel parameter given by:

$$
\kappa=\sqrt{\frac{2 I_{c} F^{2}}{\varepsilon R T}}
$$

where $I_{c}$ is ionic strength based on (molar) concentration, $\varepsilon$ is the permittivity of the medium (solution), while other symbols have their usual meaning.

The surface charge densities of interfacial planes are related to the corresponding surface concentrations of interfacial ions: the surface charge density of the 0 -plane and the $\beta$-plane (plane where the centers of counterions are located) are denoted $\sigma_{0}$ and $\sigma_{\beta}$, respectively. The net surface charge density $\sigma_{\mathrm{s}}$ is equal in magnitude but opposite in sign to that in diffuse layer $\left(\sigma_{\mathrm{d}}\right)$ and in the case of adsorption of e.g. organic acids the net surface charge density equals to:

$$
\sigma_{\mathrm{s}}=-\sigma_{\mathrm{d}}=\sigma_{0}+\sigma_{\mathrm{a}}
$$

where $\sigma_{\mathrm{a}}$ presents the surface charge due to the adsorbed ions. According to the Gouy-Chapman theory, in the case of (1:1) symmetrical electrolytes the relationship between the surface charge densities $\left(\sigma_{\mathrm{d}}, \sigma_{\mathrm{s}}\right)$ and the electrostatic potential at the onset of the diffuse layer $\Psi_{\mathrm{d}}$ for planar geometry is given by:

$$
\sigma_{\mathrm{d}}=-\sigma_{\mathrm{s}}=-\sqrt{8 R T \varepsilon I_{c}} \sinh \left(F \Psi_{\mathrm{d}} / 2 R T\right)
$$

or

$$
\Psi_{\mathrm{d}}=-\frac{2 R T}{F} \operatorname{ar} \sinh \frac{\sigma_{\mathrm{d}}}{\sqrt{8 R T \varepsilon I_{c}}}
$$

According to the general model, the (inner) Helmholtz layer could be considered as a capacitor with two planes; 0-plane and $\beta$-plane. The inner layer capacitance $C_{1}$ is assumed to be constant and is commonly defined as:

$$
C_{1}=\frac{\sigma_{0}}{\Psi_{0}-\Psi_{\beta}}
$$

In the literature various values of $C_{1}$ could be found. For example, Sverjensky ${ }^{10}$ predicted the triple layer model capacitances to be between 0.5 and $1.55 \mathrm{~F}$ $\mathrm{m}^{-2}$ for various metal oxide electrolyte interfaces. Shimizu and coworkers ${ }^{11,12}$ calculated the surface-areanormalized capacitance values for hematite electrode/electrolyte $\left(\mathrm{NaCl}\right.$ and $\left.\mathrm{NH}_{4} \mathrm{Cl}\right)$ solution interface to be between 1 and $1.4 \mu \mathrm{F} \mathrm{cm}^{-2}$. These values are at least one order of magnitude smaller than those obtained e.g. by fitting the potentiometric titration data of iron oxide powders. On the other hand, in the case of specific adsorption of different cations and anions higher capacitance values are found. For specific adsorption of $\mathrm{Cd}(\mathrm{II})$ ions on hematite Chibowski and Janusz ${ }^{13}$ obtained the capacitance of $2.4 \mathrm{~F} \mathrm{~m}^{-2}$. 


\section{EXPERIMENTAL}

\section{Materials}

All solutions were prepared using deionised water $(\kappa<3$ $\left.\mu \mathrm{S} \mathrm{cm}{ }^{-1}\right)$. Chemicals used were: $\mathrm{HClO}_{4}(70 \%, \rho \approx 1.67$ $\mathrm{g} \mathrm{cm}^{-3}, M_{\mathrm{r}}=100.46$, Riedel de Haën $), \mathrm{NaOH}(0.1 \mathrm{~mol}$ $\mathrm{dm}^{-3}$, Sigma-Aldrich), $\mathrm{NaClO}_{4}\left(\geq 99 \%\right.$ p.a., $M_{\mathrm{r}}=$ 122.44, Sigma-Aldrich), maleic acid (p.a, $M_{\mathrm{r}}=116.07$, Kemika Zagreb), fumaric acid (synthesized at Laboratory of Organic Chemistry, Department of Chemistry, Faculty of Science, University of Zagreb), standard buffers $\mathrm{pH}=3$ (Kemika Zagreb), $\mathrm{pH}=7$ (SigmaAldrich), $\mathrm{pH}=10$ (Sigma-Aldrich). Hematite particles were a product of Alfa, Johnson Matthey $\mathrm{GmbH}$ (Karslruhe, Germany) having a specific surface area of $8.8 \mathrm{~m}^{2} \mathrm{~g}^{-1}$ and diameter around $500 \mathrm{~nm}$. The particles were used without further purification.

The electrode was made from a hematite single crystal ( $\mathrm{SCr}$ ) (origin: Vesuvius, Italy). The properties and construction of the $\mathrm{SCr}$ electrode were described in more details earlier. ${ }^{8}$ After surface potential measurement (in the presence of maleic or fumaric acid) the crystal plane was cleaned by exposure of electrode to $\mathrm{NaOH}$ solution $\left(c \approx 1 \times 10^{-3} \mathrm{~mol} \mathrm{dm}{ }^{-3}\right)$ and was sonicated during 1-2 minutes. Procedure was repeated with deionized water instead $\mathrm{NaOH}$ solution. After sonication, electrode was washed again with deionized water. The electric resistance of the SCr-hematite electrode was measured directly and was approximately $7 \mathrm{M} \Omega$.

\section{Methods}

\section{Batch Adsorption Experiments}

Adsorption of maleic and fumaric acid on hematite was studied as a function of $\mathrm{pH}$ at $25^{\circ} \mathrm{C}$. Suspensions of hematite particles were prepared by the following procedure: hematite was weighed in the glass tubes which were then filled with $15 \mathrm{~mL}$ of suspension solution so that the final mass concentration of hematite was $15 \mathrm{~g}$ $\mathrm{dm}^{-3}$. The samples were prepared by adding appropriate volume of stock solution of maleic or fumaric acid, 0.1 mol dm ${ }^{-3}$ perchloric acid and $0.1 \mathrm{~mol} \mathrm{dm}^{-3} \mathrm{NaOH}$ to adjust the $\mathrm{pH}$ value and then filled with water up to 25 $\mathrm{ml}$. $\mathrm{pH}$ of suspension solution was measured with combined electrode with reference $\mathrm{Ag}|\mathrm{AgCl}| 3 \mathrm{M} \mathrm{KCl}$ (Metrohm 6.0234.100) electrode and 827 Metrohm pHmeter. Suspensions were stirred for 90 minutes and then left overnight so the adsorption equilibrium and separation of the two phases can be achieved. Before filtration of suspensions, equilibrium $\mathrm{pH}$ was measured. Suspensions were filtered with Filtrak 390 filter paper with porosity 3-5 $\mu \mathrm{m}$ (Spezialpapierfabrik Niederschlag, Germany). Equilibrium concentration of maleic and fumaric acid was calculated from absorbance measured with Cary UV-Vis Spectrophotometer, Varian. Before spectrophotometric measurements all samples were acidified with $\mathrm{HClO}_{4}$ to a $\mathrm{pH} \approx 0.5$ to protonate the acids (> $90 \%$ ), i.e. to convert them to $\mathrm{H}_{2} \mathrm{~A}$ form. $\mathrm{Ab}$ sorbance values used in the calculation of maleic concentration were measured at $\lambda=206 \mathrm{~nm}$ and those of fumaric acid were measured at $\lambda=210 \mathrm{~nm}$.

\section{Electrokinetic Measurements}

The electrokinetic (zeta) potential of hematite particles was measured before and after adsorption of dicarboxylic acids by means of a ZetaPlus Zeta Potential Analyser, Brookhaven Instruments Corporation at $25.0^{\circ} \mathrm{C}$. The instrument uses electrophoretic light scattering and the Laser Doppler Velocimetry method for determination of particle velocity. Zeta potential was calculated from mobility values using the Smoluchowski equation. The measurements in the presence of maleic or fumaric acid were performed after taking $1.5 \mathrm{ml}$ of each supernatant solution and mixing it with small amount of hematite particles because initial concentration of hematite $\left(15 \mathrm{~g} \mathrm{dm}^{-3}\right)$ was too high for electrokinetic measurements. Zeta potential of hematite particles in absence of respective acids was measured for suspension of he-matite particles $\left(\gamma=100 \mathrm{mg} \mathrm{dm}^{-3}\right)$ in $1 \times 10^{-3} \mathrm{~mol} \mathrm{dm}^{-3} \mathrm{HClO}_{4}$ after setting $\mathrm{pH}$ values of aliquots with $0.1 \mathrm{~mol} \mathrm{dm}^{-3} \mathrm{NaOH}$ solution. The experiments were performed at $1 \times 10^{-3} \mathrm{~mol} \mathrm{dm}^{-3}$ ionic strength (i.e. concentration of perchloric acid).

\section{Surface Potential Measurements}

The potential of the hematite single crystal electrode was measured using the Metrohm $713 \mathrm{pH}$-meter. The $\mathrm{pH}$ was measured with a glass electrode (Metrohm 6.0123.100) using a separate Metrohm $713 \mathrm{pH}$-meter. The common reference electrode was $\mathrm{Ag}|\mathrm{AgCl}| 3 \mathrm{M} \mathrm{KCl}$, (Metrohm 6.0729.100) with a salt bridge filled with 0.1 mol dm${ }^{-3} \mathrm{NaClO}_{4}$. The glass electrode was calibrated with three standard buffers. In the course of measurements the system was thermostated at $25.0^{\circ} \mathrm{C}$ and kept under argon atmosphere. In order to determine the electrode potential of hematite in perchloric acid without added maleic or fumaric acid, perchloric acid solution ( $30 \mathrm{~mL}, c=1 \times 10^{-3} \mathrm{~mol} \mathrm{dm}^{-3}$ ) was titrated with the base $\left(c=0.1 \mathrm{~mol} \mathrm{dm}^{-3}, \mathrm{NaOH}\right)$. The backward titration (with perchloric acid, $c=0.1 \mathrm{~mol} \mathrm{dm}^{-3}$ ) was also performed. The same kind of the cyclic titration was performed in perchloric acid solution $\left(c=1 \times 10^{-3} \mathrm{~mol} \mathrm{dm}^{-3}\right)$ in presence of maleic or fumaric acid $\left(c=1 \times 10^{-3} \mathrm{~mol} \mathrm{dm}^{-3}\right)$. The system was gently stirred with a magnetic stirrer.

\section{RESULTS}

\section{Adsorption Measurements}

Figure 1 displays the effect of the $\mathrm{pH}$ on the surface concentration of fumaric and maleic acid on hematite. Typical adsorption isotherm profile consistent with adsorption of singly $(-1)$ charged species is obtained. In 
both sets of experiments the highest adsorbed amount was observed at $\mathrm{pH} \approx 4$ which corresponds to the maximum in the speciation diagram (Figure 2) in the case of both investigated dicarboxylic acids. Therefore, in the further interpretation of data the assumption that singly charged species are adsorbed will be taken into account.

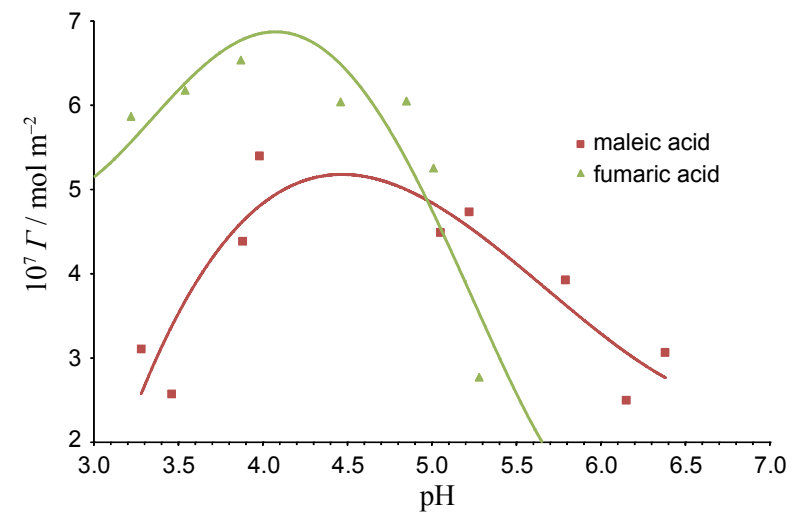

Figure 1. The effect of $\mathrm{pH}$ on the surface concentration of maleic $(\square)$ and fumaric $(\triangle)$ acid on hematite at ionic strength of $1 \times 10^{-3} \mathrm{~mol} \mathrm{dm}^{-3} ; \gamma=15 \mathrm{~g} \mathrm{dm}^{-3},\left[\mathrm{H}_{2} \mathrm{~A}\right]_{\text {in }}=1 \times 10^{-3} \mathrm{~mol} \mathrm{dm}^{-3}$, $t=25^{\circ} \mathrm{C}$.
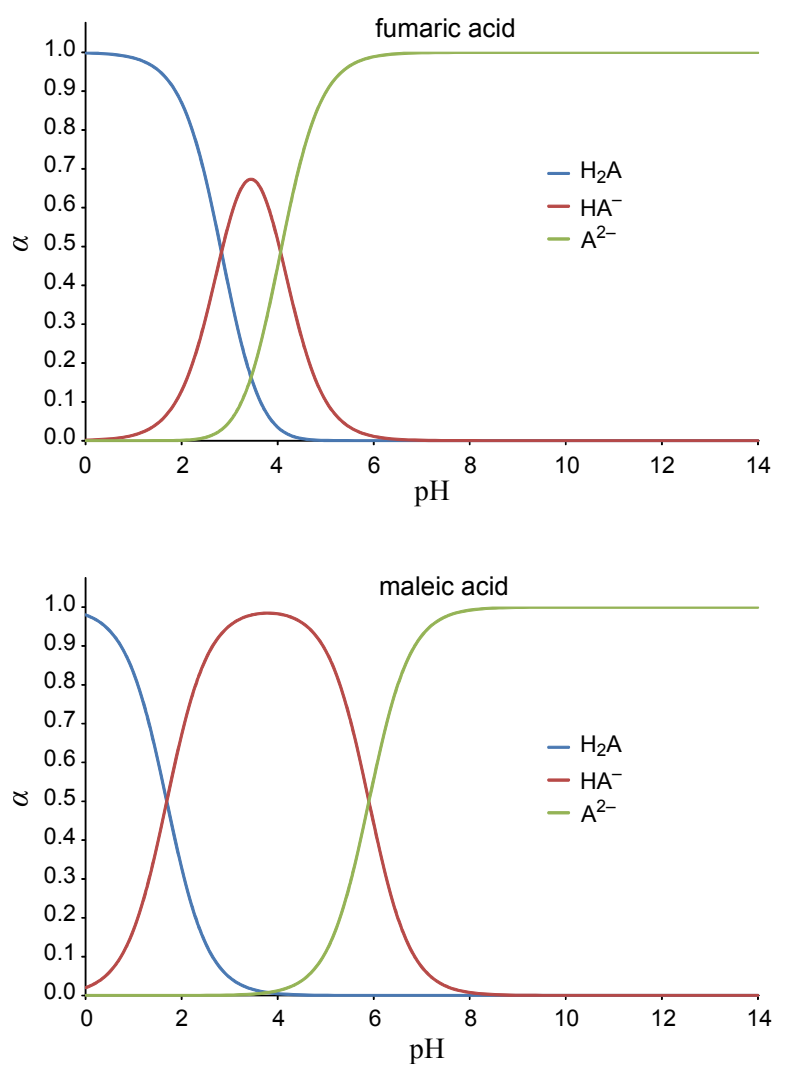

Figure 2. The speciation of fumaric and maleic acid in the bulk of the solution. In the calculations the following values of the dissociation equilibrium constants were used: ${ }^{14}$ maleic acid: $\mathrm{p} K_{1}=1.69, \mathrm{p} K_{2}=5.90$; fumaric acid: $\mathrm{p} K_{1}=2.83, \mathrm{p} K_{2}=$ 4.06, $\left[\mathrm{H}_{2} \mathrm{~A}\right]_{\text {in }}=1 \times 10^{-3} \mathrm{~mol} \mathrm{dm}^{-3}$.

\section{Electrokinetic Measurements}

In Figure 3 the influence of $\mathrm{pH}$ on the zeta potential of hematite in the absence and in the presence of maleic and fumaric acid is shown. In the absence of dicarboxylic acids (pure hematite dispersion) the isoelectric point of hematite is at $\mathrm{pH}_{\text {iep }}=6.4$ which is in accordance with literature values. ${ }^{15}$ In the presence of dicarboxylic acids $\mathrm{pH}_{\text {iep }}$ is shifted, as expected for the adsorption of negatively charged species, to the lower $\mathrm{pH}$ values being $\mathrm{pH}_{\text {iep }}=4.3$ for hematite/maleic acid and $\mathrm{pH}_{\text {iep }}=4.1$ for hematite/fumaric acid system. From these results it could be concluded that the adsorption of fumaric acid shifts the isoelectric point of pure hematite slightly more than the adsorption of maleic acid.

\section{Surface Potential Measurements}

Surface potential measurements were performed in order to elucidate the nature of binding of maleic and fumaric acid to hematite surface. The electrode potential of $\mathrm{SCr}$ hematite electrode, $E$, was measured in perchloric acid solution in the absence and in the presence of maleic and fumaric acid as function of $\mathrm{pH}$. The experiments were performed as acid-base titration (perchloric acid solution was titrated with sodium hydroxide solution and the same solution was then titrated with perchloric acid). The hysteresis for alkalimetric titration, i.e. titration with base, and acidimetric titration, i.e. titration with acid was observed, which is the result of the slow equilibration at the interface.

From the measured electrode potentials of the hematite single crystal electrode surface potentials $\Psi_{0}$ were obtained ${ }^{16}$ by

$$
\Psi_{0}=E-E_{\mathrm{cal}}
$$

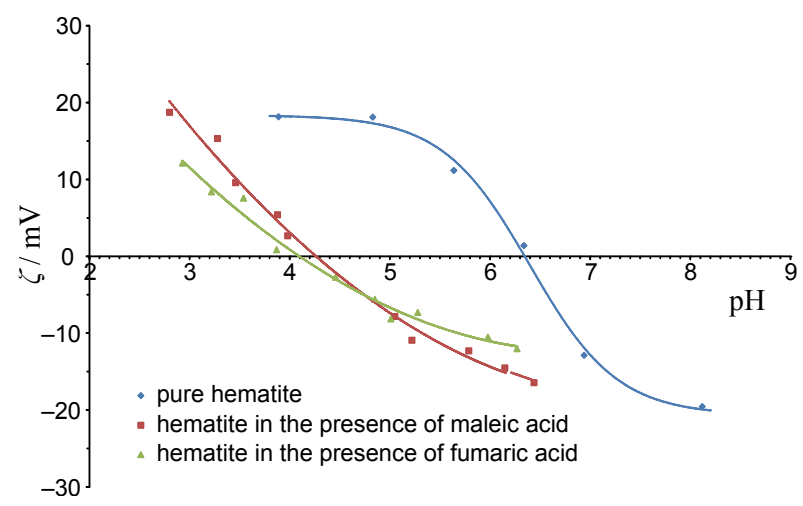

Figure 3. Electrokinetic (zeta) potential of hematite particles $\left(\gamma=100 \mathrm{mg} \mathrm{dm}^{-3}\right)$ as function of $\mathrm{pH}$ in perchloric acid solution $\left(c=1 \times 10^{-3} \mathrm{~mol} \mathrm{dm}^{-3}\right)$ before $(\diamond)$ and after adsorption of maleic $(\square)$ or fumaric acid $(\Delta)\left(c=1 \times 10^{-3} \mathrm{~mol} \mathrm{dm}^{-3}\right)$. $\mathrm{pH}$ value of suspension was changed by adding sodium hydroxide solution $\left(0.1 \mathrm{~mol} \mathrm{dm}^{-3}\right)$ at $25.0{ }^{\circ} \mathrm{C}$. 
where the value of $E_{\mathrm{cal}}$ includes all potential differences in the measuring circle, except the one at the crystal/solution interface. Once the value of $E_{\text {cal }}$ is known, surface potentials can easily be obtained from the measured electrode potentials via Equation (7). In fact, one sets the zero value of surface potential at $\mathrm{pH}_{\mathrm{pzp}}$ which was approximated by the isoelectric point $\mathrm{pH}_{\text {iep }}$ of pure hematite (determined from electrokinetic measurements to be $\mathrm{pH}_{\text {iep }}=\mathrm{pH}_{\mathrm{pzp}}=6.4$ ) assuming that at low ionic strength the point of zero potential coincides with the isoelectric point. The same value of $\mathrm{pH}_{\mathrm{pzp}}=6.4$ was used as the basis for calculation of surface potentials in the presence of maleic and fumaric acid, since it is known $^{17}$ that association of various ions shifts $\mathrm{pH}_{\text {iep }}$ more significantly than $\mathrm{pH}_{\mathrm{pzp}}$, and $E_{\mathrm{cal}}$ value was calculated accordingly. In Figure 4 surface potential values determined in the abovementioned way are presented as arithmetic mean of surface potentials calculated from both titration curves (alkalimetric and acidimetric) for all three examined cases. The surface potential of hematite is found to decrease with $\mathrm{pH}$, indicating that the surface becomes less positively or more negatively charged. The slope of the $\Psi_{0}(\mathrm{pH})$ function is found to be lower than the Nernstian slope. ${ }^{17,18}$

From the results shown in Figure 4 it could be concluded that the presence of maleic and fumaric acid influences the surface potentials in similar manner and that no significant difference in the curves obtained in the presence and in the absence of fumaric acids was observed. Taking into account these results, as well as the results of zeta potential measurements, it could be assumed that it is more probable that adsorption of dicarboxylic acids takes place in the $d$ - and not in the 0 -plane. Therefore, in the interpretation of the data the assumption of the adsorption in the d-plane will be used.

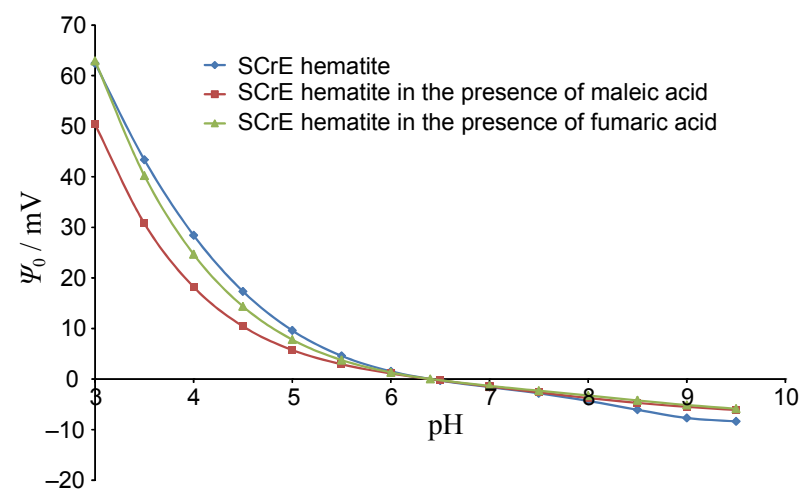

Figure 4. The dependence of surface potential $\left(\Psi_{0}\right)$ of hematite on $\mathrm{pH}$ in perchloric acid solution $\left(c=1 \times 10^{-3} \mathrm{~mol} \mathrm{dm}^{-3}\right)$ in the absence $(\diamond)$ and in the presence of maleic $(\square)$ and fumaric $(\Delta)$ acid at $25^{\circ} \mathrm{C}$. Equilibration time: $20 \mathrm{~min}$. Surface potential values were calculated from electrode potentials via Equation (7).

\section{INTERPRETATION}

The interpretation of the experimental data, performed according to the Surface Complexation Model, was based on the following procedures:

$$
\begin{aligned}
& \zeta \stackrel{l_{e}}{\longrightarrow} \Psi_{\mathrm{d}} \stackrel{\text { G.C. }}{\longrightarrow} \sigma_{\mathrm{s}}\left(=-\sigma_{\mathrm{d}}\right) \stackrel{-z F \Gamma_{\mathrm{a}}}{\longrightarrow} \sigma_{0} \\
& \Psi_{0} \stackrel{\sigma_{0}}{\longrightarrow} C_{1}
\end{aligned}
$$

The first step in the interpretation was the calculation of $\Psi_{\mathrm{d}}$ from the measured $\zeta$-potential (Equation 1) assuming different values of the distance between d-plane and electrokinetic slip plane $l_{e}$ in the range from 0 to $25 \AA$ by step of $5 \AA$. No significant effect of the choice of $l_{\mathrm{e}}$ value on the final results was found and therefore the value of $l_{\mathrm{e}}=5 \AA$ was used in further calculations. Surface charge density $\sigma_{\mathrm{s}}\left(=-\sigma_{\mathrm{d}}\right)$ was then calculated from $\Psi_{\mathrm{d}}$ potential via Equation (4).

In the next step of the interpretation procedure one calculates $\sigma_{0}$ from $\sigma_{\mathrm{s}}$ and $\sigma_{\mathrm{a}}$, where $\sigma_{\mathrm{a}}$ is equal to $z F \Gamma_{\mathrm{a}}$ ( $\Gamma_{\mathrm{a}}$ presents the adsorbed amount of maleic or fumaric acid as obtained from adsorption experiments, Figure 1). The charge number $z$ was taken to be -1 which is in accordance with adsorption results (see the comparison with speciation).

Now, taking into account $\sigma_{0}$ values obtained via the procedure (8), in the parallel procedure (9) the value of capacitance $C_{1}$ could be estimated from the measured $\Psi_{0}$ values and from calculated $\sigma_{0}$ values. In that way the capacitance $C_{1}$ was estimated to be relatively high, i.e. $2.2 \pm 0.3 \mathrm{~F} \mathrm{~m}^{-2}$ for both hematite/maleic and hematite/fumaric acid aqueous interface. Since the value of $C_{1}$ is a specific characteristics of species bound to the surface it is not surprising that examined acids behave differently with respect to e.g. inorganic counterions. ${ }^{10-13}$ The obtained results are also in accordance with the results we obtained earlier for cadmium/goethite aqueous interface. ${ }^{19}$

In the final step of the interpretation procedure the equilibrium constants of adsorption were calculated for both dicarboxylic acids taking into account the assumptions that adsorbed ions are located in d-plane (deduced from zeta and surface potential measurements) and that charge number of adsorbed species is -1 (deduced from adsorption measurements). Therefore, we used the modified Langmuir isotherm ${ }^{5}$ in the form

$$
\frac{1}{\Gamma}=\frac{1}{\Gamma_{\max }}+\frac{1}{K^{\circ} \exp \left(-z \psi_{\mathrm{d}} F / R T\right) \Gamma_{\max } c_{\text {eq }}}
$$

where $c_{\mathrm{eq}}$ is the equilibrium concentration of adsorbable species. 
From the interpretation based on Equation (10) the value of maximum adsorbed amount $\Gamma_{\max }$ was estimated to be $5.2 \times 10^{-7} \mathrm{~mol} \mathrm{~m}^{-2}$ and $6.3 \times 10^{-7} \mathrm{~mol} \mathrm{~m}^{-2}$ for maleic and fumaric acid, respectively. Hwang ${ }^{20}$ also obtained higher maximum adsorbed amount for fumaric acid $\left(1.62 \times 10^{-6} \mathrm{~mol} \mathrm{~m}^{-2}\right.$ at $\left.\mathrm{pH}=4.1\right)$ than for maleic acid $\left(1.41 \times 10^{-6} \mathrm{~mol} \mathrm{~m}^{-2}\right.$ at $\left.\mathrm{pH}=4.2\right)$. Such a difference between the maximum adsorbed amounts obtained in these two studies could be due to the various hematite samples (i.e. different method of preparation, particle size and isoelectric point).

The corresponding thermodynamic equilibrium constants are determined as $\log K^{\circ}=4.0 \pm 0.1$ and $\log K^{\circ}=4.9 \pm 0.2$ for adsorption of maleic and fumaric acid, respectively.

\section{CONCLUSION}

The comparison of experimental results obtained from adsorption, zeta potential and surface potential measurements of pure hematite with the corresponding measurements after adsorption of maleic and fumaric acid could give valuable information about the parameters at hematite/dicarboxylic acid aqueous interface. No significant difference in the surface potential values of single crystal hematite electrode with and without the presence of dicarboxylic acids in the examined $\mathrm{pH}$ region was observed. On the other hand, the adsorption of both maleic and fumaric influenced the zeta potential values. The simultaneous interpretation of adsorption, zeta potential and surface potential data on the basis of the Surface Complexation Model leads also to the values of the parameters in the electrical interfacial layer such as equilibrium constant of adsorption and the capacitance $C_{1}$. The obtained results suggest that simultaneous interpretation of adsorption, surface and zeta potential measurements could give valuable information about the investigated electrical interfacial layer.

Acknowledgements. This research was supported by the Ministry of Science, Education and Sports of the Republic of Croatia (project No. 119-119342-2961) and by the COST Action
CM1101. The authors are grateful to Tajana Preočanin for helpful discussions and Danijel Namjesnik for technical assistance.

\section{REFERENCES}

1. E. D. Vega and P. A. Colinas, J. Argent. Chem. Soc. 97 (2009) 195-206.

2. J. Rosenqvist, K. Axe, S. Sjöberg, and P. Persson, Colloids Surf. A: Physicochem. Eng. Aspects 220 (2003) 91-104.

3. H.-L. Yao and H.-H. Yeh, Langmuir 12 (1996) 2989-2994.

4. Y.-S. Hwang and J. J. Lenhart, Langmuir 24 (2008) 13934 13943.

5. D. Kovačević, N. Kallay, I. Antol, A. Pohlmeier, H. Lewandowski, and H-D. Narres, Colloids Surf. A 140 (1998) 261-267.

6. D. Kovačević, A. Pohlmeier, G. Özbas, H-D. Narres, and N. Kallay, Colloids Surf. 166 (2000) 225-233.

7. D. Kovačević, D. Mazur, T. Preočanin, and N. Kallay, Adsorption 16 (2010) 405-412.

8. N. Kallay, Z. Dojnović, and A. Čop, J. Colloid Interface Sci. 286 (2005) 610-614.

9. D. Kovačević, T. Preočanin, S. Žalac, and A. Čop, Croat. Chem. Acta 80 (2007) 287-301.

10. D. A. Sverjensky, Geochim. Cosmochim. Acta 65 (2001) 3643 3655 .

11. K. Shimizu, A. Shchukarev, and J.-F. Boily, Abstract \#1589, $221^{\text {st }}$ ECS Meeting, Seattle, 2012.

12. K. Shimizu, A. Lasia, and J.-F. Boily, Langmuir 28 (2012) 7914-7920.

13. S. Chibowski and W. Janusz, Applied Surface Sci. 196 (2002) 343-355.

14. J. J. Lenhart, R. Heyler, E. M. Walton, and S. E. Mylonet, J. Colloid Interface Sci. 345 (2010) 556-560.

15. M. Kosmulski, Surfactant Science Series, Vol. 145: Surface Charging and Points of Zero Charge, Boca Raton: CRC Press, 2009.

16. N. Kallay, T. Preočanin, D. Kovačević, J. Lützenkirchen, and E. Chibowski, Croat. Chem. Acta 83 (2010) 357-370.

17. N. Kallay, T. Preočanin, and T. Ivšić, J. Colloid Interface Sci. 309 (2007) 21-27.

18. T. Preočanin, A. Selmani, D. Mazur, and N. Kallay, Applied Surf. Sci. 256 (2010) 5412-5415.

19. D. Kovačević, A. Pohlmeier, G. Özbas, H.-D. Narres and N. Kallay, Progr. Colloid Polym. Sci. 112 (1999) 183-187.

20. Y. S. Hwang, Adsorption of naturally-occurring dicarboxylic acids at the hematite/water interface, Dissertation, The Ohio State University, 2008. 